\title{
PNEUMOCOCCAL DISEASE
}

\section{WHAT IS PNEUMOCOCCAL DISEASE?}

Pneumococcal disease is caused by infection with the bacteria Streptococcus pneumonia. This infection can cause a range of illnesses, the most common including:

- pneumonia (infection of the lungs);

- otitis media (infection of the middle ear);

- meningitis (infection of the membranes around the brain).

Other bacteria can also cause these diseases.

\section{WHAT ARE THE SYMPTOMS?}

Symptoms depend on the site of infection and the age of the person. They may include:

- pneumonia - shortness of breath, fast breathing, fever, lack of energy, loss of appetite, headache, chest pain, and cough;

- otitis media-(especially in children less than three years old, usually after a cold or flu-like illness) crying, tugging at the ear, fever, irritability, poor hearing, and sometimes diarrhoea and vomiting;

- meningitis-fever, headache, stiff neck, nausea, vomiting, and drowsiness.

\section{HOW IS IT SPREAD?}

The bacteria often live harmlessly in the throat of healthy people. They are passed from person-to-person via droplets when coughing or sneezing, kissing, or indirectly via toys and other soiled items. However, it is uncommon for people who are exposed to the bacteria to become ill.

\section{WHO IS MOST AT RISK?}

People most at risk for the infection include:

- Aboriginal and Torres Strait Islander people;

- the elderly;

- children under two years of age;

- people with other medical conditions such as lung disease, heart disease, cancer, kidney disease, HIV infection, or malnutrition;

- people whose spleen has been removed or doesn't work properly.

Outbreaks have occurred in childcare centres, nursing homes, and other institutions.

Pneumococcal infections are more common in Winter and early Spring.

\section{HOW IS IT DIAGNOSED AND TREATED?}

Your doctor can diagnose pneumococcal disease by listening to your symptoms, giving you a physical examination, and doing some tests. Tests may include a chest $\mathrm{x}$-ray, and taking samples to look for the bacteria in the infected part of your body (for example: sputum, blood or cerebrospinal fluid).

Treatment may include:

- antibiotics;

- medicine to control the fever and pain, such as paracetamol;

- fluids to prevent dehydration;

- rest.

\section{HOW IS IT PREVENTED?}

\section{Children}

A vaccine to prevent pneumococcal disease in small children was introduced in Australia in 2001. In New South Wales it is recommended for:

- all Aboriginal and Torres Strait Islander children up to two-years of age;

- children under five years of age who:

- were born with certain immune deficiencies;

- are receiving certain drugs or radiation treatment;

- have spleens that don't work properly;

- have HIV infection;

- have certain kidney diseases;

- have heart disease causing cyanosis or heart failure;

- have cerebrospinal fluid leaks.

For babies less than six months of age, three doses of vaccine are given two months apart. Fewer doses are needed for older children.

\section{Adults and older children}

A vaccine for preventing pneumococcal disease in adults and older children has been available for many years. Vaccination is recommended for:

- all people aged 65 years and older;

- Aboriginal and Torres Strait Islander people aged 50 years or older;

- people without a working spleen;

- people who are immunocompromised;

- people with chronic illnesses (for example: heart, kidney, lung, diabetes, or alcohol related illnesses);

- patients with cerebrospinal fluid leaks.

Re-vaccination is recommended every five years.

In some people at highest risk of severe pneumococcal disease, preventive antibiotic treatment may also be recommended.

For further information please contact your local public health unit, community health centre, or doctor.

March 2002 